\title{
A Coal Mine Multi-Point Fiber Ethylene Gas Concentration Sensor
}

\author{
Yubin WEI ${ }^{1,2}$, Jun CHANG ${ }^{1 *}$, Jie LIAN ${ }^{1}$, and Tongyu LIU $^{2}$ \\ ${ }^{1}$ School of Information Science and Engineering and Shandong Provincial Key Laboratory of Laser Technology and \\ Application, Shandong University, Jinan, 250100, China \\ ${ }^{2}$ Laser Research Institute of Shandong Sciences Academy, Jinan, 250014, China \\ *Corresponding author: Jun CHANG～E-mail: changjun@sdu.edu.cn
}

\begin{abstract}
Spontaneous combustion of the coal mine goaf is one of the main disasters in the coal mine. The detection technology based on symbolic gas is the main means to realize the spontaneous combustion prediction of the coal mine goaf, and ethylene gas is an important symbol gas of spontaneous combustion in the coal accelerated oxidation stage. In order to overcome the problem of current coal ethylene detection, the paper presents a mine optical fiber multi-point ethylene concentration sensor based on the tunable diode laser absorption spectroscopy. Based on the experiments and analysis of the near-infrared spectrum of ethylene, the system employed the $1.62 \mu \mathrm{m}$ (DFB) wavelength fiber coupled distributed feedback laser as the light source. By using the wavelength scanning technique and developing a stable fiber coupled Herriot type long path gas absorption cell, a ppm-level high sensitivity detecting system for the concentration of ethylene gas was realized, which could meet the needs of coal mine fire prevention goaf prediction.
\end{abstract}

Keywords: Spontaneous combustion, signature gas, ethylene, tunable diode laser absorption spectroscopy (TDLAS), distributed feedback laser (DFB), Herriott cell

Citation: Yubin WEI, Jun CHANG, Jie LIAN, and Tongyu LIU, "A Coal Mine Multi-Point Fiber Ethylene Gas Concentration Sensor ," Photonic Sensors, 2015, 5(1): 67-71.

\section{Introduction}

Mine fire is one of the main disasters in coal mines, and $56 \%$ coal mines in China had spontaneous combustion disasters, among them, the goaf spontaneous combustion possessed a great proportion. According to statistics, China's state-owned key coal mines with spontaneous combustion dangerous mines accounted for about $51.3 \%$ of the total. Mine fire may influence the safety in production, even burning coal resources and materials equipment, causing casualties, and even leading to gas or coal dust explosion. Therefore, goaf coal spontaneous combustion prediction and early warning and positioning have the important significance for reducing the loss of life and property. To solve this problem of the exploitation of coal spontaneous combustion tendency, researchers have established a series of prediction technologies. The signature gases analysis method is used mainly to determine the process of coal spontaneous combustion or the development trend of coal spontaneous combustion according to the gas production of $\mathrm{CO}, \mathrm{C}_{2} \mathrm{H}_{2}, \mathrm{C}_{2} \mathrm{H}_{4}$, and alkane. The ethylene gas is one of the important signature gases generated in the process of the coal accelerated

Received: 25 February 2014 / Revised version: 12 May 2014

(C) The Author(s) 2014. This article is published with open access at Springerlink.com

DOI: $10.1007 / \mathrm{s} 13320-014-0206-\mathrm{Z}$

Article type: Regular 
oxidation stage, which has the important significance for the spontaneous combustion prediction. At present, generally the tube pipe monitoring analysis system based on the gas chromatography is adopted, which has the disadvantages of equipment complex, long sampling and analysis cycle, long distance sample pipeline leak easily leading to inaccurate measurement, and the urgent need to improve the performance $[1,2]$.

TDLAS is a high-sensitivity, real-time, and dynamic trace gas detection technique. Owing to the high monochromaticity of the laser diode, the absorption spectra of gases can be detected using an isolated absorption line of each gas molecule. In this way, different molecules can be identified while avoiding interference from other spectra. According to this technique, the near-infrared waveband is matched with the low-loss window of an optical fiber, and by using such optical fibers, the remote transport of a light beam is realized [3-6]. Moreover, the remote, on-line, and real-time detection of the gas concentration is achieved by combining TDLAS and optical fiber sensing techniques. Owing to the whole system being based on an optical fiber, the sensor is able to enter the goaf to carry out field tests, instead of pumping out gases, thus avoiding measurement errors during pipe sampling [9].

To overcome the problems with current ethylene detection methods used in the coal mine, a mine-used, multi-point optical fiber-based sensor for detecting the ethylene gas concentration was presented based on the TDLAS approach. On the basis of experiments and analysis of ethylene using

$$
I(\lambda)=I_{0}(\lambda) \exp [-\alpha(\lambda) C L]=I_{0}(\lambda) \exp [-P S(T) \varphi(\lambda) C L]
$$

where $\alpha(\lambda)$ is the absorption coefficient of the gas; $L[\mathrm{~cm}]$ is the length of the gas absorbing light; $S(T)\left[\mathrm{cm}^{-2} \cdot \mathrm{atm}^{-1}\right]$ is the intensity of the characteristic spectral line of the gas, which represents the absorption intensity of the spectral lines which is related to temperature; $P[\mathrm{~atm}]$ is the total pressure of the gas; $C$ is the volume concentration of the gas; $\phi(\lambda)[\mathrm{cm}]$ is a line-shape the near-infrared spectroscopy, the fiber coupling distributed feedback laser (DFB) in the near-infrared waveband at a wavelength of $1.62 \mu \mathrm{m}$ was chosen as the light source. Furthermore, a wavelength scanning technique was used, and a long optical path length based gas cell was developed with a stable and reliable fiber coupling using the Herriot principle. Using this gas cell, the ethylene concentration could be detected at ppm-level high sensitivity. It provides a novel detection method for the forecasting of spontaneous combustion in the coal goaf.

\section{Test principle}

In different conditions, the wavelength, intensity, and polarization, etc of molecules absorption or emission spectra and the material structure characteristic have their intrinsic relationships. Different gas molecule structures correspond to different absorption spectra; the same gas with different concentrations in the same absorption peak position has different absorption intensities, so by detecting the gas absorption intensity on the specific wavelength light, the composition and concentration of gas can be determined.

According to the Lambert-Beer law, when a beam of parallel light with an intensity $I_{0}(\lambda)$ passes through a gas cell filled with the gas under test and provided that the spectrum of the light source encompasses one or more of the absorption lines of the gas, the relationship among the transmitted light intensity $I(\lambda)$, incident intensity $I_{0}(\lambda)$, and the gas concentration $C$ is $[7,8]$

function, which represents the shape of the absorption spectral lines which is associated with the temperature, total pressure, and proportional composition of the gas. The most commonly used line-shape functions are those: Lorentz, Gauss, and Voigt functions. After taking logarithm of (1), the integration over the whole frequency domain is 


$$
P C S(T) L=\int_{-\infty}^{\infty}-\ln \left(\frac{I}{I_{0}}\right) d \lambda=A .
$$

The gas concentration is therefore calculated from

$$
C=\frac{\int_{-\infty}^{\infty}-\ln \left(\frac{I}{I_{0}}\right) d \lambda}{P S(T) L}=\frac{A}{P S(T) L} .
$$

Given that parameters such as the pressure, line intensity, and length of the gas absorbing light path are known, the integral value of $-\ln \left(I / I_{0}\right)$ in the frequency domain is substituted into (3), and the final gas concentration can then be obtained [10].

\section{Absorption lines of ethylene gas in the near-infrared region}

Under normal detection conditions, the moisture and carbon dioxide contents in air are the most important sources of interference with gas measurement. To avoid cross-interference from other gases, the absorption lines are required to be carefully selected, and they can be directly obtained from the high-resolution transmission molecular absorption database (HITRAN), as shown in Fig. 1 where the intrinsic absorption peak of ethylene lies near $3.3 \mu \mathrm{m}$. However, there are no absorption data for ethylene at $1.7 \mu \mathrm{m}$ in the HITRAN database.

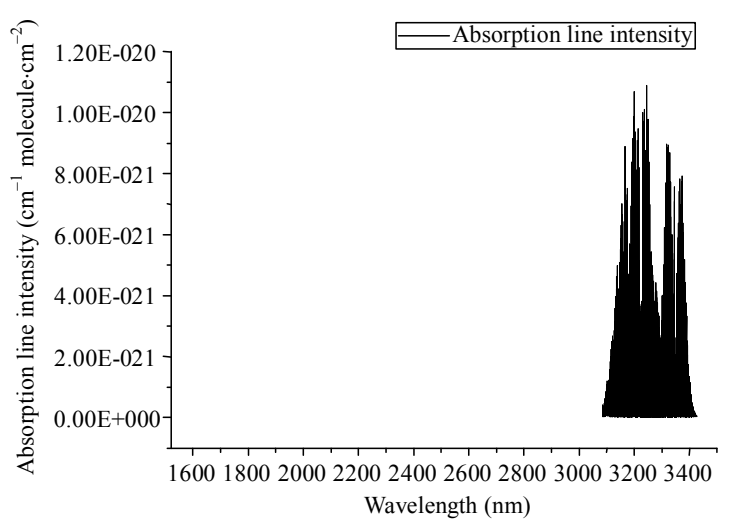

Fig. 1 Absorption lines of ethylene in the near-infrared region $(1.5 \mu \mathrm{m}$ to $3.5 \mu \mathrm{m})$.

The authors found the absorption lines of ethylene at around $1.7 \mu \mathrm{m}$ from the PNNL database (Fig. 2). However, an accurate and complete absorption spectrum for ethylene remains to be found.
To obtain accurate absorption lines, the standard ethylene gas was tested under broadband light at $1650 \mathrm{~nm}$ (range: $1550 \mathrm{~nm}$ to $1700 \mathrm{~nm}$ ), at an ethylene gas concentration of $20 \%$, using a gas cell with an optical path of $10 \mathrm{~cm}$ across its absorption cavity, and with the absorption spectrum data obtained by an AQ6370 spectrometer. The gas cell was firstly cleaned using the pure nitrogen gas, and then the transmittance signal in the clean gas cell was tested using the light source. Afterwards, the ethylene gas was injected into the gas cell to detect the absorbed transmittance signal. After normalization, the absorption lines of the ethylene gas were acquired. As shown in Fig. 3, ethylene presented a strong absorption at $1626 \mathrm{~nm}$, and there were strong absorption bands at approximately $1620 \mathrm{~nm}$ and $1630 \mathrm{~nm}$. They could be used as signature absorption lines. In the proposed system, the absorption line at $1620 \mathrm{~nm}$ was chosen as the determining wavelength to avoid cross-absorption of interference gases, because of its preferable line-type and high absorption intensity. Above all, this wavelength lay at the edge of the L-waveband in optical communications, and a laser device able to operate at this wavelength was easily obtained and cheap.

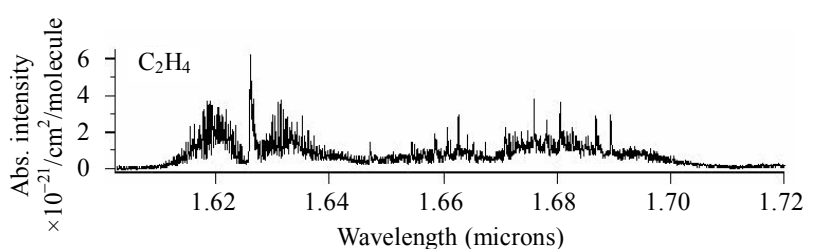

Fig. 2 Absorption lines of ethylene in the near-infrared region (PNNL database).

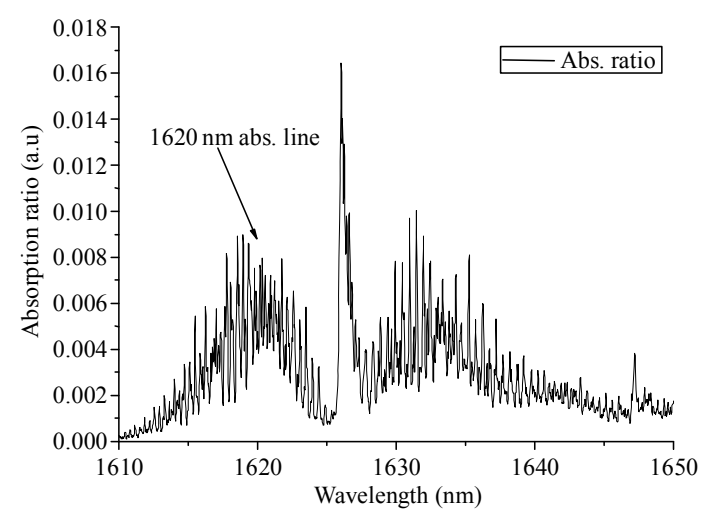

Fig. 3 Absorption lines of ethylene in the infrared region. 


\section{Optical fiber ethylene sensor system: experimental work}

The structure of the proposed optical fiber-based ethylene monitoring system is shown in Fig. 4. The DFB laser device was driven by a current-driving circuit, and its working temperature was controlled by a temperature control circuit. Then, the laser device output a continuous spectrum with a central wavelength of $1620 \mathrm{~nm}$ to realize wavelength modulation. The gas cells were $9.3 \mathrm{~m}$ in length. An InGaAs photoelectric detector was employed to encompass the range from $600 \mathrm{~nm}$ to $1700 \mathrm{~nm}$. Three PIN detectors PD1, PD2, and PD3 were used to detect the output intensity of the light after passing through the gas cell, the light intensity of the output signal of the light source after passing through two couplers, and the output intensity of the light after passing through the reference gas cell, respectively. The reference gas cell was filled with $\mathrm{C}_{2} \mathrm{H}_{4}$ gas at $100 \%$ concentration to find the position of the peak absorption. Afterwards, three detection signals were collected using a data acquisition unit and then normalized. The normalized signal was subjected to a least square fit against the standard signal to calculate the concentration of $\mathrm{C}_{2} \mathrm{H}_{4}$ gas [11].

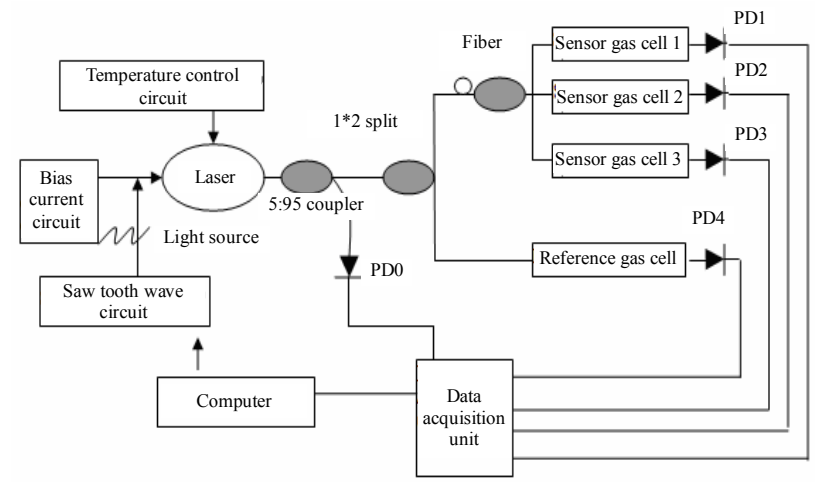

Fig. 4 Structure of the optical fiber ethylene monitoring system.

The software based on LabVIEW 8.0 was programmed to collect data, display results in real time, store data, set the over-limit alarm value, and process the least squares fitting algorithm, etc. The real-time display showed the concentration of the gas under test: all data were stored in an SQL-server database at different pre-set time intervals.

In the experiment, we used nitrogen gas as the background gas. Then, pure nitrogen gas and signature ethylene gases at concentrations of $20 \mathrm{ppm}$, $79.8 \mathrm{ppm}, 120 \mathrm{ppm}$, and $150 \mathrm{ppm}$ were injected into the gas cells, respectively. To eliminate the system and optical noises produced in the Herriot cell, we collected the signal after 100 times average. Figure 5 shows the ethylene gas absorption signals at different concentrations.

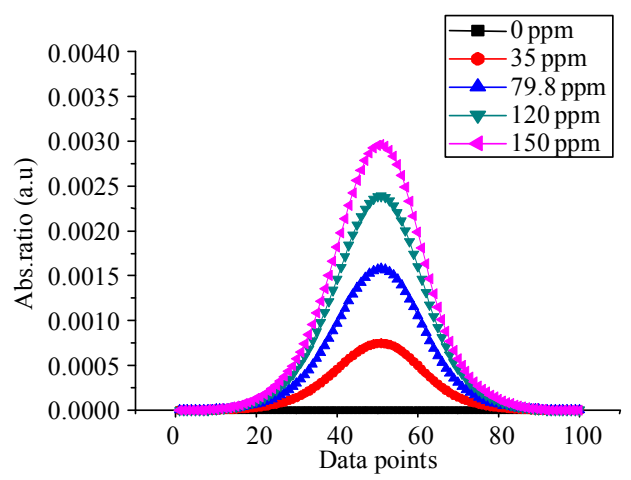

Fig. 5 Spectrum absorption curves for five unknown gas concentrations.

Figure 6 shows that the absorption ratio was well correlated with the concentration of the signature gas. The linear relationship between the gas concentration and absorptivity is

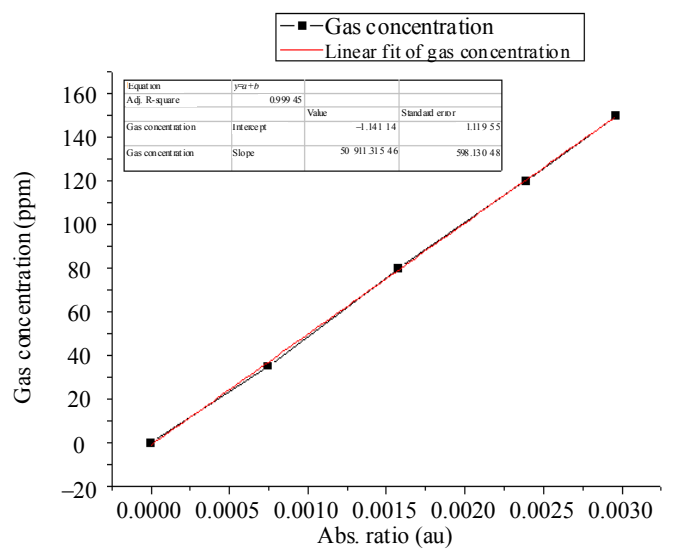

Fig. 6 Absorption ratio versus concentration of signature gas.

$$
y=50911.3155 x-1.1411
$$

with an R-square of 0.9996 and a standard error of 1.12. Equation (4) is put into software, then verified using an ethylene gas concentration of $20 \mathrm{ppm}$. 
Figure 7 shows that the error lay within $\pm 2 \mathrm{ppm}$ and was very stable. By calculating a five-point moving average across the calculated concentration data, the concentration stability of final detection was shown to be less than $1 \mathrm{ppm}$, which met the accurate requirements for fire forecasting in coal mines.

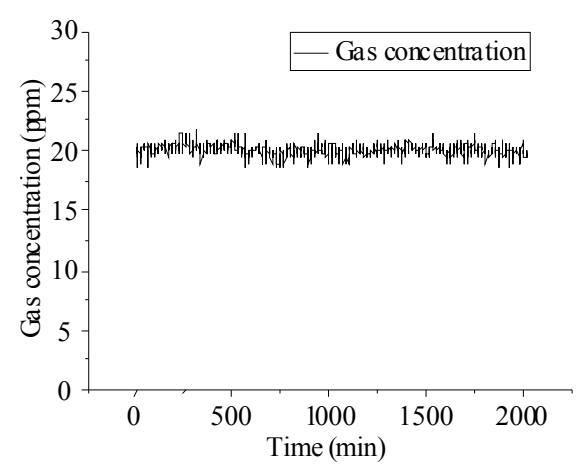

Fig. 7 Long-term stability of signature ethylene at a concentration of $20 \mathrm{ppm}$.

\section{Conclusions}

In order to solve the problem of ethylene online detection for the prediction of coal spontaneous combustion, the paper presents a mine optical fiber multi-point ethylene concentration sensor based on the tunable diode laser absorption spectroscopy. Based on the experiments and analysis of the near-infrared spectrum of ethylene, the system employed the $1.62 \mu \mathrm{m}$ (DFB) wavelength fiber coupled distributed feedback laser as the light source. By using the wavelength scanning technique and with the development of a stable fiber coupled Herriot type long path gas absorption cell, a ppm-level high sensitivity detecting system for the concentration of ethylene gas was realized, which can meet the needs of coal mine goaf fire prediction and provides a new means for the prediction of spontaneous combustion of the coal mine goaf.

\section{Acknowledgement}

This work was supported by the National Natural Science Foundation of China (60977058 \& 61475085) and Fundamental Research Funds of
Shandong University (2014YQ011).

Open Access This article is distributed under the terms of the Creative Commons Attribution License which permits any use, distribution, and reproduction in any medium, provided the original author(s) and source are credited.

\section{Reference}

[1] X. Xian, H. Wang, D. Jiang, and B. Liu, "China coal mine fire fighting technology research," Engineering Science, 2001, 3(12): 28-32.

[2] H. Luo and Y. Liang, "Status and prospects of coal spontaneous combustion prediction technology," China Safety Science Journal, 2003, 13(3): 76-80.

[3] N. Li, "Research on gas detection and 2D distribution reconstruction by tunable diode laser absorption spectroscopy technique," Ph.D. dissertation, Zhejiang University, China, 2008.

[4] J. Reid and D. Labrie, "Second harmonic detection with tunable diode lasers-comparision of experiment and theory," Applied Physics B, 1981, 26(3): 203-210.

[5] J. M. Supple, E. A. Whittaker, and W. Lenth, "Theoretical description of frequency modulation and wavelength modulation spectroscopy," Applied Optics, 1994, 33(27): 6294-6302.

[6] X. Tu, W. Liu, Y. Zhang, F. Dong, M. Wang, T. Wang, et al., "Second-harmonic detection with tunable diode laser absorption spectroscopy of $\mathrm{CO}$ and $\mathrm{CO}_{2}$ at $1.58 \mu \mathrm{m}$," Spectroscopy and Spectral Analysis, 2006, 26(7): 1190-1194.

[7] Y. Shang, Y. Wei, C. Wang, T. Lei, and Z. Song, "The research of acetylene gas concentration on-line monitoring system based on absorption spectra," Chinese Journal of Sensors and Actuators, 2010, 23(2): 171-174.

[8] S. Zhang, Q. Wang, Y. Zhang, F. Song, K. Chen, G. chou, et al., "Water vapor detection system based on scanning spectra," Photonic Sensors, 2012, 2(1): 71-76.

[9] D. S. Borns, D. C. Hovde, S. Chen, and J. A. Sivler, "Early fire sensing using near IR diode laser spectroscopy," in Proc. SPIE, vol. 4817, pp. 73-81, 2002.

[10] G. Lv, J. Chang, Q. Wang, Q. Wang, and W. Wei, "Research progress of optical $\mathrm{H}_{2} \mathrm{O}$ sensor with a DFB diode laser," Photonic Sensors, 2014, 4(2): 113-119.

[11] D. P. Leleux, R. Claps, W. Chen, F. K. Tittel, and T. L. Harman, "Applications of Kalman filtering to real-time trace gas concentration measurements," Applied Physics B, 2002, 74(1): 85-93. 